\title{
Dynamic left ventricular dyssynchrony and severe mitral regurgitation caused by exercise: should we go beyond the guidelines?
}

This article was published in the following Dove Press journal: International Medical Case Reports Journal

\author{
Emilie Laflamme' \\ François Philippon' \\ Kim O'Connor' \\ Jean-François Sarrazin' \\ Vincent Auffret ${ }^{\prime}$ \\ Vincent Chauvette \\ Michelle Dubois ${ }^{2}$ \\ Pierre Voisine ${ }^{3}$ \\ Sébastien Bergeron' \\ Mario Sénéchal' \\ 'Department of Cardiology, \\ ${ }^{2}$ Research Center, ${ }^{3}$ Department of \\ Cardiovascular Surgery, Institut \\ Universitaire de Cardiologie et \\ de Pneumologie de Québec, Laval \\ University, Quebec City, QC, Canada
}

Correspondence: Mario Sénéchal Institut universitaire de cardiologie et de pneumologie de Québec, Laval University, 2725 Chemin Ste-Foy,

Quebec, QC GIV 4G5, Canada

Tel + I $41865687 \mid$ I

Fax +I 4186568157

Email mario.senechal@criucpq.ulaval.ca

\begin{abstract}
Guidelines for cardiac resynchronization therapy (CRT) have been established, but there may be a subgroup of patients not identified in these guidelines who may benefit from this therapy. We report a patient with a dynamic left ventricular dyssynchrony and severe mitral regurgitation caused by exercise successfully treated with CRT. Exercise testing should be considered in patients with left ventricular ejection fraction $<35 \%$ and QRS $<130 \mathrm{~ms}$ with severe heart failure symptoms that are unexplained by rest echocardiography evaluation in order to rule out ischemia and/or dynamic left ventricular dyssynchrony. In the presence of exerciseinduced left ventricular bundle branch block, the implantation of CRT should be contemplated. Keywords: cardiac resynchronization therapy, mitral regurgitation, left ventricular dyssynchrony, heart failure, exercise
\end{abstract}

\section{Plain language summary}

Guidelines for cardiac resynchronization therapy (CRT) have been established, but there may be a subgroup of patients not identified in these guidelines who may benefit from this therapy. We report a patient with a dynamic left ventricular dyssynchrony and severe mitral regurgitation caused by exercise successfully treated by CRT.

\section{Introduction}

Cardiac resynchronization therapy (CRT) is an established therapy for symptomatic heart failure (HF). In the most recent Canadian Cardiovascular Society (CCS)/Canadian Heart Rhythm Society (CHRS) guidelines, indications for CRT are as follows: patients in sinus rhythm with a left bundle branch block (LBBB) $\geq 130 \mathrm{~ms}$, ejection fraction $\leq 35 \%$, and who remain in New York Heart Association (NYHA) functional class II, III, or ambulatory class IV despite optimal medical therapy. It is also recognized that patients with a QRS duration $>150 \mathrm{~ms}$ benefit the most from CRT. ${ }^{1}$ ACCF/AHA/HRS guidelines were updated in 2012, showing Class IIa indication for CRT in patients with left ventricular ejection fraction (LVEF) $<35 \%$ and LBBB with a QRS duration between 120 and $149 \mathrm{~ms}$ with Class II, III, or ambulatory class IV symptoms despite optimal medical therapy. ${ }^{2}$ In patients with chronic HF and a QRS duration $<120 \mathrm{~ms}$ or in the presence of exercise-induced LBBB, CRT is not recommended. Responders to CRT have been identified, but there may be a subgroup of patients who may benefit from this therapy despite not being represented in clinical trials. ${ }^{4}$ In this case report, we present a patient with a dynamic LV dyssynchrony and severe mitral regurgitation (MR) caused by exercise who presented with multiple episodes of pulmonary edema. He was 
successfully treated by CRT. This case report was written with the agreement of the Institut universitaire de cardiologie et de pneumologie de Québec direction, and the patient provided written informed consent for the case details to be published.

\section{Case description}

A 69-year old patient was first evaluated at the Institut universitaire de cardiologie et de pneumologie de Québec in 2009 for shortness of breath. Past medical history included peripheral vascular disease, asthma, and dyslipidemia. The initial investigation led to a percutaneous revascularization procedure for significant coronary disease. He presented over the next 2 years with various episodes of angina that required further stenting, and he finally had quadruple coronary artery bypass grafting in 2011. A year later, despite optimal medical therapy, the patient remained in NYHA functional class III and experienced multiple acute pulmonary edema episodes after light exercise that required mechanical ventilation twice. After surgery, the patient was followed at our HF clinic regularly (every 2 months) and medication was up-titrated as tolerated (bisoprolol $10 \mathrm{mg}$ daily, ramipril $10 \mathrm{mg}$ twice daily, furosemide $40 \mathrm{mg}$ twice daily, and spironolactone $25 \mathrm{mg}$ daily). Blood pressure was well controlled, and no cardiac arrhythmia was documented. A thallium stress test demonstrated significant viability in all myocardial segments without residual ischemia. The electrocardiogram showed sinus rhythm with incomplete LBBB (QRS interval of 116 ms, Figure 1). The echocardiography showed a 35\% LVEF (LV end diastolic and systolic diameter of $65 \mathrm{~mm}$ and 53 $\mathrm{mm}$ respectively), a mild functional MR with an effective regurgitant orifice area (EROA) of $10 \mathrm{~mm}^{2}$, and right ventricle systolic pressure (RVSP) at $24 \mathrm{mmHg}$ plus central venous pressure. He underwent a bicycle ergometer test to evaluate MR under dynamic conditions. Exercise tolerance was poor, with less than $50 \mathrm{~W}$ reached in 5:30 minutes. The echocardiography performed during exercise showed a 35\% LVEF and a gradual worsening of MR from mild to severe with an EROA of $38 \mathrm{~mm}^{2}$ at peak stress. The RVSP reached $55 \mathrm{mmHg}$ plus central venous pressure, and the QRS interval during the exercise widened from $116 \mathrm{~ms}$ to $135 \mathrm{~ms}$ (Figure 2). Based on multiple acute HF episodes, poor exercise tolerance, presence of dynamic MR and the widening of the QRS $>130 \mathrm{~ms}$ at peak stress, a CRT was implanted. After CRT implantation, the patient remained free from acute HF episodes and his functional class improved (NYHA II). Medication remained similar, but furosemide was decreased to $40 \mathrm{mg}$ daily. In January 2015, he performed another bicycle ergometer. Exercise tolerance improved significantly at 9:45 minutes (125 W) and he remained asymptomatic. The rest LVEF was $40 \%$ (LV end diastolic diameter and LV end systolic diameter of $54 \mathrm{~mm}$ and $42 \mathrm{~mm}$, respectively) and reached $45 \%$ at peak exercise without ischemia. From rest to maximal exercise, MR went from mild to moderate (EROA $15 \mathrm{~mm}^{2}$ ) and the RVSP increased from $24 \mathrm{mmHg}$ to $45 \mathrm{mmHg}$.

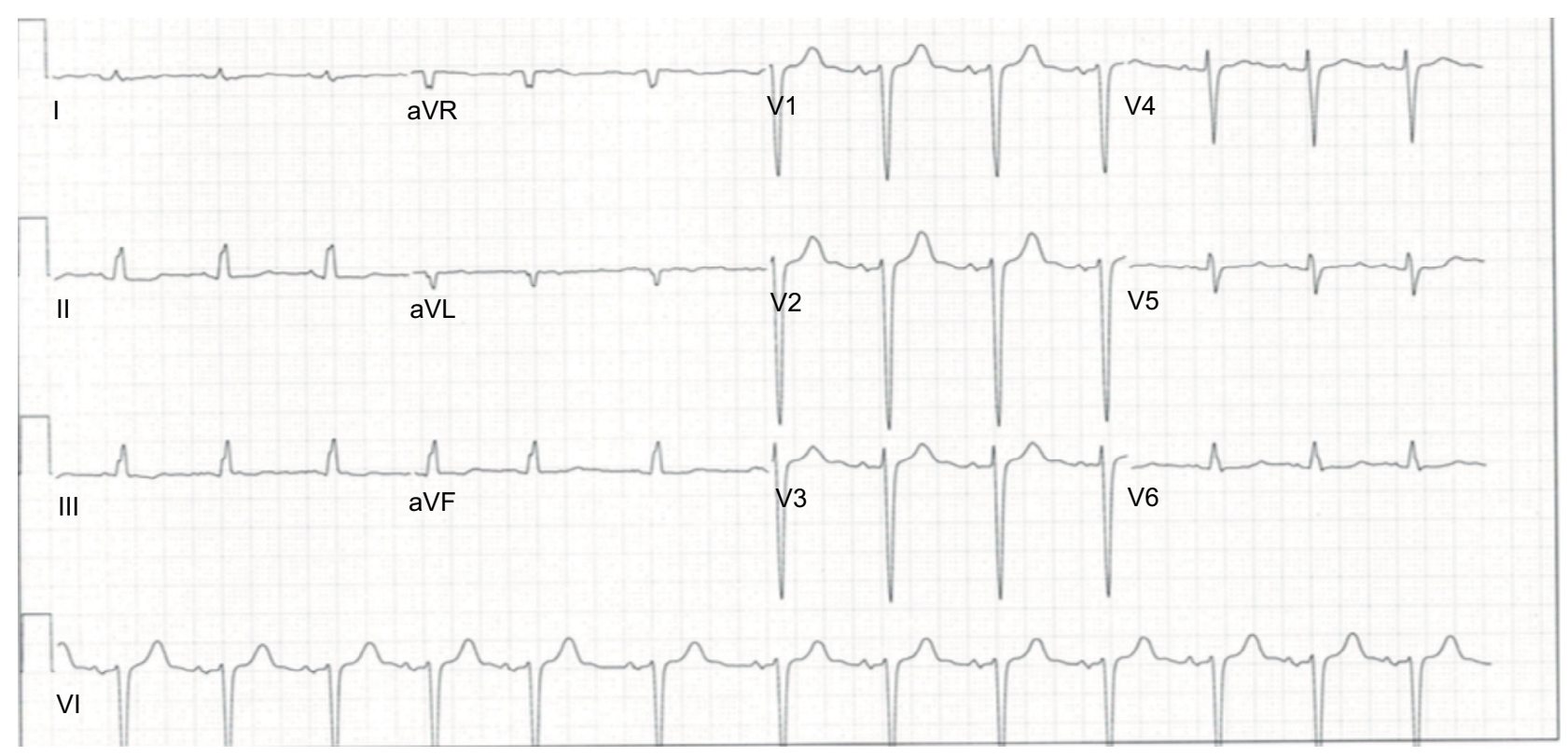

Figure I ECG (QRS II6 ms) at rest before CRT.

Abbreviations: CRT, cardiac resynchronization therapy; ECG, echocardiogram. 


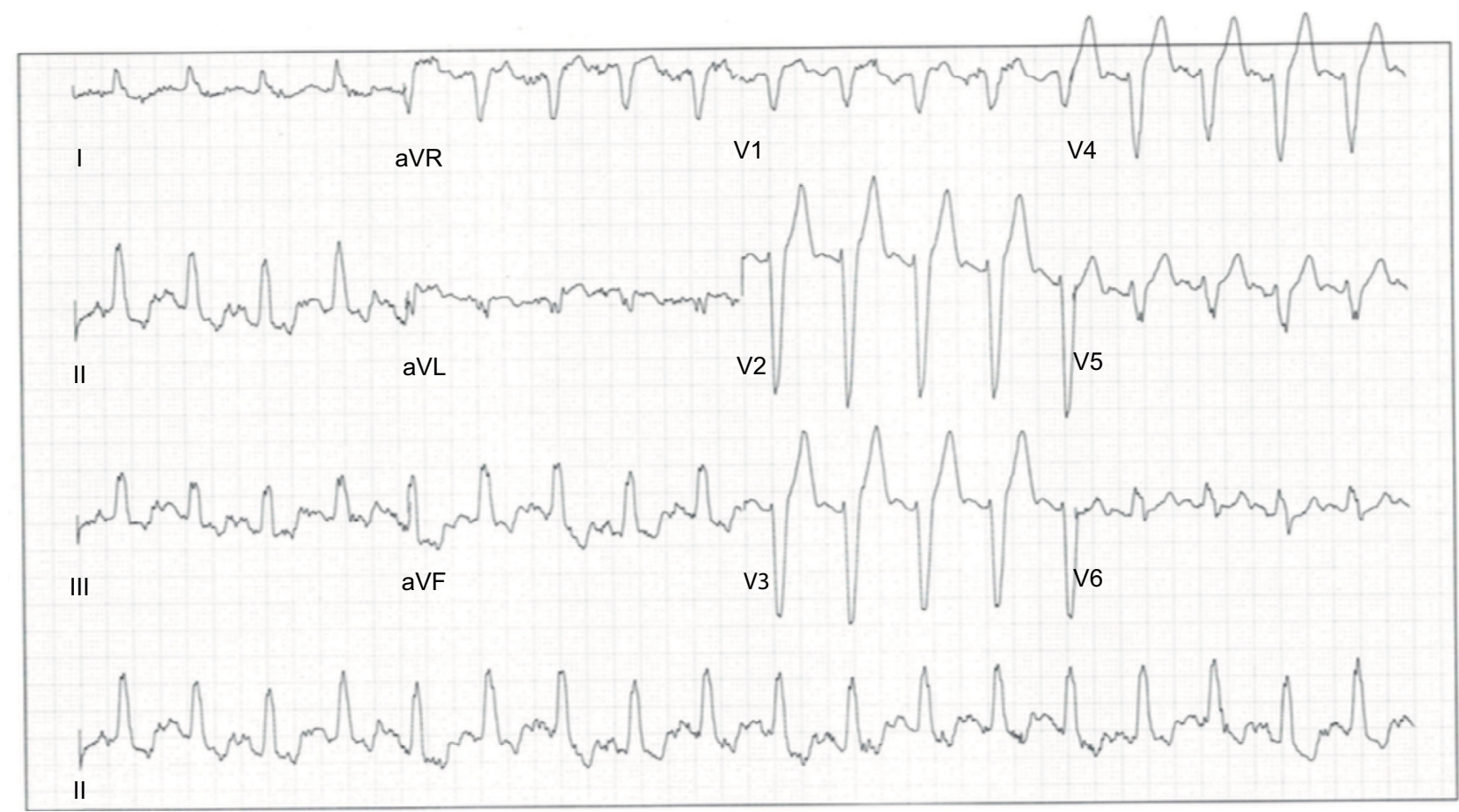

Figure 2 ECG (QRS $135 \mathrm{~ms}$ ) demonstrating LBBB during stress before CRT.

Abbreviations: CRT, cardiac resynchronization therapy; ECG, echocardiogram; LBBB, left bundle branch block.

\section{Discussion}

The annual incidence of LBBB is about $10 \%$ in ambulatory patients with LV systolic dysfunction and chronic HF. ${ }^{1}$ Based on current guidelines criteria, only a small proportion of patients with HF are indicated for CRT. Moreover, in the RETHINQ trial and in the recent randomized double-blind LESSER-EARTH trial, there was no evidence of CRT benefits in patients with $\mathrm{HF}$ and a QRS $<120 \mathrm{~ms}$, and morbidity was not favorably modified by CRT. ${ }^{1}$ However, despite those studies, our case describes a patient who might benefit from CRT without fulfilling traditional guidelines criteria. The prevalence of exercise-induced LBBB is approximately $0.4 \%$ $1.1 \%$. It is usually associated with adverse prognosis, higher mortality, and major cardiovascular events. ${ }^{3}$ The etiology of exercise-induced LBBB is unclear, but its development could be related to myocardial ischemia and/or conduction abnormality in response to increasing heart rate. Both ischemia and aberrant conduction can precipitate LV dyssynchrony, leading to reduced cardiac output and high LV filling pressure with or without increase in functional MR manifesting in shortness of breath or eventually in pulmonary edema. Of note, our patient required multiple intubations because of severe pulmonary edema which seemed to be induced by light exercise. The widening of QRS >130 ms with dynamic MR at peak stress echocardiography pre-CRT and the reduction of MR at peak stress echocardiography post-CRT suggest that both exercise LBBB and dynamic MR may have contributed to the patient's symptomatology. ${ }^{3}$ Increased tenting area resulting from LV remodeling has been identified as a determinant of the severity of resting and exercise functional MR. Besides reducing cardiac performance, myocardial dyssynchrony may decrease mitral valve closing forces, thereby exacerbating functional MR during exercise in patients with HF. In a previous manuscript, D'Andrea et $\mathrm{al}^{4}$ included 60 patients with idiopathic dilated cardiomyopathy and narrow QRS $(<120$ $\mathrm{ms})$. Cardiac synchronicity measured between the onset of QRS complex and the peak myocardial systolic velocity at peak exercise demonstrated a significant electromechanical delay (dyssynchrony) in $58 \%$ of patients. In $78 \%$ of patients, exercise induced an increased mitral EROA. Piérard and Lancellotti $^{5}$ demonstrated that patients who presented acute pulmonary edema were more likely to present a dynamic MR, therefore suggesting an important pathological role of exercise-induced MR in acute HF episodes. The absence of association between rest and exercise EROA in MR has previously been demonstrated, enlightening the importance of stress evaluation. The dynamic modifications in MR are mostly driven by geometrical distortion in LV configuration 
and after load under stress. ${ }^{4}$ Moreover, LV contractility is clearly influenced by the presence of widening QRS/increased asynchrony at stress in a subgroup of patients with HF. It is already known that CRT might improve rest MR. Ennezat et $\mathrm{al}^{6}$ found that CRT reduced $\mathrm{MR}$ at rest but also prevented the increase of EROA and regurgitant volume during exercise. They observed that positive impact on dynamic MR might be related with an improvement in contractility, which increases in transmitral force closure. This report concerns a patient with narrow QRS complex who experienced an acute exacerbation of HF after light exercise. Increase of heart rate induced acceleration-dependent LBBB with severe functional MR followed by acute HF necessitating mechanical ventilation twice. Exercise testing evaluation pre- and post-CRT implantation and the favorable long-term clinical evolution without recurrence of pulmonary edema suggest that CRT may have prevented the acute consequences of the exerciseinduced LBBB in this patient. Patients with mild HF, narrow QRS complex, and episodes of severe HF following stress may benefit from exercise testing. It seems reasonable to rule out acceleration-dependent LBBB with LV dyssynchrony in these patients before excluding them from CRT. ${ }^{7}$

\section{Conclusion}

Our case report depicted a patient with severe HF symptoms and repetitive life-threatening pulmonary edema episodes. Exercise testing with or without echocardiography should be considered in patients with an LVEF $<35 \%$ and QRS $<130$ ms with severe HF symptoms that are unexplained by rest echocardiography evaluation in order to rule out ischemia and dynamic LV dyssynchrony. In the presence of exercise-induced LBBB, the implantation of CRT should be contemplated.

\section{Disclosure}

The authors report no conflicts of interest in this work.

\section{References}

1. Exner DV, Birnie DH, Moe G, et al. Canadian Cardiovascular Society guidelines on the use of cardiac resynchronization therapy: evidence and patient selection. Can J Cardiol. 2013;29(2):182-195.

2. Epstein AE, DiMarco JP, Ellenbogen KA, et al. 2012 ACCF/AHA/HRS Focused Update Incorporated Into the ACCF/AHA/HRS 2008 Guidelines for Device-Based Therapy of Cardiac Rhythm Abnormalities: A Report of the American College of Cardiology Foundation/American Heart Association Task Force on Practice Guidelines and the Heart Rhythm Society. J Am Coll Cardiol. 2013;61(3):e6-e75.

3. Czuriga D, Lim PO. Cardiac resynchronization therapy relieves intractable angina due to exercise-induced left bundle branch block without left ventricular systolic dysfunction: a detailed case study. J Cardiovasc Electrophysiol. 2016;27:609-612.

4. D'Andrea A, Caso P, Cuomo S, et al. Effect of dynamic myocardial dyssynchrony on mitral regurgitation during supine bicycle exercise stress echocardiography in patients with idiopathic dilated cardiomyopathy and 'narrow' QRS. Eur Heart J. 2007;28(8):1004-1011.

5. Piérard LA, Lancellotti $P$. The role of ischemic mitral regurgitation in the pathogenesis of acute pulmonary edema. $N$ Engl $J$ Med. 2004;351:1627-1634.

6. Ennezat PV, Gal B, Kouakam C, et al. Cardiac resynchronisation therapy reduces functional mitral regurgitation during dynamic exercise in patients with chronic heart failure: an acute echocardiographic study. Heart. 2006;92:1091-1095.

7. Zeppenfeld K, Schalij MJ, Bleeker GB, Holman ER, Bax JJ. Acceleration-dependent left bundle branch block with severe left ventricular dyssynchrony results in acute heart failure: are there more patients who benefit from cardiac resynchronization therapy? J Cardiovasc Electrophysiol. 2006;17:101-103.
International Medical Case Reports Journal

Publish your work in this journal

The International Medical Case Reports Journal is an international, peer-reviewed open-access journal publishing original case reports from all medical specialties. Previously unpublished medical posters are also accepted relating to any area of clinical or preclinical science. Submissions should not normally exceed 2,000 words or

\section{Dovepress}

4 published pages including figures, diagrams and references. The manuscript management system is completely online and includes a very quick and fair peer-review system, which is all easy to use. Visit http://www.dovepress.com/testimonials.php to read real quotes from published authors. 